\title{
Facility location via fuzzy modeling and simulation
}

\author{
Mahmood Rezaei ${ }^{1 *}$, M.H. Fazel Zarandi ${ }^{2}$ \\ ${ }^{1}$ HEC Management School, University of Liège, 14 Rue Louvrex, 4000 Liège, Belgium \\ ${ }^{2}$ Department of Industrial Engineering, Amirkabir University of Technology, Hafez Av., \\ Tehran, Iran
}

\begin{abstract}
This paper presents a continuous facility location model with fuzzy methodology. The developments concern mainly to some drawbacks in the initial model which takes it far from being used in practice. A fuzzy modeling method is proposed to estimate the required functions in the initial model. Structure identification in the proposed fuzzy modeling method is carried out using subtractive clustering, and parameter identification is conducted via some heuristics as well as an optimization problem.

Furthermore, a simulation method along with some heuristic relations is used for implementation and evaluation of the modified model. Efficiency of the proposed method to fuzzy modeling as well as the proposed simulation method is presented by a numerical example.
\end{abstract}

Keywords: Facility location; fuzzy modeling; fuzzy rule base; simulation.

\section{Introduction}

In the domain of supply chain management there are two complementary issues for most production systems: facility layout and facility location. Facility layout problems deal with the position of manufacturing machines, stores, and manpower inside a firm. It has attracted the attention of many researchers because it can considerably reduce the material handling costs and yet increase flexibility of the manufacturing system. Facility layout is usually regarded as an optimization problem to determine the most efficient layout based on some prespecified criteria. $[1,12]$

Facility location, on the other hand, concerns the choice of the location of one or multiple facilities, in a given geographical space and subject to some constraints, to optimally fulfill predetermined objectives. It is a strategic decision making compared to facility layout which is more operational. Facility location might be part of a more

\footnotetext{
${ }^{*}$ Corresponding author. Tel: +32-4-2327342; Fax: +32-4-2327240.
} 
comprehensive problem named Production-Distribution System Design (PDSD). In general, a PDSD problem involves the determination of the best configuration, regarding location and size of the facilities and distribution centers, their technology content, commodity offerings, and transportation decisions, for achieving a firm's long term goals [11]. It is a strategic issue for both industrial firms and governmental agencies, in that an efficient PDSD can result in reducing transportation costs, enforcing operational efficiency and logistic performance, and improving the quality of the services.

Facility location problems are usually categorized based on some characteristics such as: number of facilities (single/multiple), objective function (single/multiple), solution space (discrete/network/continuous), number of commodities (single/multiple), capacity limitation (yes/no), shape of facility (point/extensive), and demand (discrete/continuous). However, it should be noted that these characteristics are not restricted to the above mentioned ones $[7,8,14,19,20]$.

The main decision variables in facility location problems are coordination (geographical location) of each facility. Besides, number of facilities, capacity of each facility, and its respective service region might be decision variables of the model as well. In problems with discrete solution space, the facilities can conceptually be placed only at a limited number of eligible points on the plane or in a network. On the other hand, in a continuous solution space the points to be located can generally be placed anywhere on the plane or in a network $[18,20]$.

Typically, continuous location problems tend to be nonlinear optimization problems, while discrete location problems involve zero-one variables that result in integer programming optimization problems. Moreover, considering the number of facilities and the capacity of each facility as the decision variables makes the model more complicated, from both the problem formulation and the problem solving perspectives. As a result, the most complex facility location problems are those that consider the number of facilities as well as their coordination and capacities as decision variables in a continuous solution space with multiple objectives and multiple commodities.

Although the field of facility location is active from the research point of view, when it comes to applications, there appears to be a significant deficit, at least as compared to other similar fields [20]. One reason for this gap could be that many applications cannot be solved by the plain version of a location problem, but further constraints (e.g. forbidden regions) must be introduced in order to construct a reasonable model [7]. To 
this end, many concepts, tools and techniques of artificial intelligence such as fuzzy logic can be used to improve the implementation of numerous models in operations research $[10,11,18,20]$.

The literature shows that the majority of the works in the area of facility location have used fuzzy theory to fuzzify the parameters of the model or have dealt with the facility location problem as a fuzzy multiple attribute decision making problem [2,3,10,16,24]. Wen and Kang [25], for example, consider fuzzy demands to construct some optimization models for facility location. Unlike these models, a novel utilization of fuzzy theory in facility location is proposed in this paper, in which we deal with estimation of some required nonlinear functions through fuzzy modeling. Although the proposed method is used to develop an existing model proposed by Dasci and Verter

[6], it can be extended to formulate any facility location model in which a mathematical function, especially a nonlinear one, is needed for demand density, transportation cost, operational cost, fixed cost, and so on.

The remaining of this paper is organized as follows: Section 2 concisely states the initial model and its drawbacks. Section 3 introduces the required materials for fuzzy modeling and the proposed method. In section 4, development of the initial model through fuzzy modeling and simulation is discussed in details. Section 5 indicates implementation of the proposed method via a numerical example. Finally, conclusions of the paper and future works are presented in section 6.

\section{Problem Statement}

Continuous models are successfully used in spatial economics and logistics, but there are probably a few papers that use continuous models for facility design [6]. Models of this type assume that customers are spread over a given market area and prescribe the optimal service region for each facility to be established [6]. Dasci and Verter [6] present a PDSD model in which the concentration is on facility location with the following main features:

1) the model consists of two multi-element layers: manufacturing facilities and end customers,

2) the number of manufacturing facilities is a decision variable,

3) the model is a single-product PDSD problem, 
4) customers' demand is deterministic and is specified by a density function,

5) there is no limitation for manufacturing facilities' capacity,

6) all customers' demand must be satisfied.

The objective is to minimize the sum of total annual costs including fixed, operational, and transportation costs. They present a modeling framework based on the use of continuous functions to represent spatial distributions of cost and costumer demand. Herein, the Dasci and Verter's model [6] is briefly addressed.

Assume that a firm wants to open some manufacturing facilities in a demand area, where each facility serves a single service region. In such a case, decision variables are: the number of facilities, the location of facilities, and the service region of each facility. Facilities can be located anywhere in the demand area. Let's $M$ denotes the market area for which the following variables and parameters are defined:

\section{Decision variables:}

$n$ : number of facilities

$\left(x_{i}, y_{i}\right)$ : location coordination of the $i$ th facility

$R_{i}$ : service region to be served by facility $i$, which is located at $\left(x_{i}, y_{i}\right) \in R_{i}$ $A_{i}$ : area of the service region $i\left(\mathrm{~km}^{2}\right)$

\section{Parameters:}

$D(x, y)$ : demand density at $(x, y) \in M\left(\right.$ item $/ \mathrm{km}^{2}$.year $)$

$F(x, y)$ : fixed cost of opening a facility at $(x, y) \in M(\$ / y e a r)$

$f(x, y, w)$ : operational cost of opening a facility of size $w$ at $(x, y) \in M$ (\$/year)

$g\left(x, y, R_{i}\right):$ total transportation cost given facility location $(x, y) \in M$ and service region $R_{i}$ (\$/year)

It is assumed that the whole demand is to be satisfied, thus service region must cover the demand area. Furthermore, each service region is served by a single facility. Generally, service regions can have irregular, rather than just geometrical, shapes. Fig. 1 depicts a hypothetical sample solution.

Fig. 1. A sample solution of the initial model.

The objective is to minimize total annual costs including annual fixed, operational, 
and transportation costs. It is assumed that all parameters vary slowly within a service region. Also, the operational cost is assumed to be a linear function as:

$$
f(x, y, w)=O(x, y) . w
$$

where, $O(x, y)$ is the production cost in the facility located in $(x, y)$ for each unit of the product and $w$ is the total annual production. As a result, the total annual operational cost is defined as:

$$
\sum_{i} f\left(x_{i}, y_{i}, w\right)=\sum_{i} f\left(x_{i}, y_{i}, \int_{R_{i}} D(x, y) d x d y\right)
$$

Since

$$
A_{i}=\int_{R_{i}} d x d y
$$

and

$$
\int_{R_{i}} D(x, y) d x d y \approx D\left(x_{i}, y_{i}\right) \cdot A_{i}
$$

Eq. (2) is transformed to:

$$
\sum_{i} f\left(x_{i}, y_{i}, w\right) \approx \sum_{i} f\left(x_{i}, y_{i}, D\left(x_{i}, y_{i}\right) \cdot A_{i}\right) \approx \sum_{i} O\left(x_{i}, y_{i}\right) \cdot D\left(x_{i}, y_{i}\right) \cdot A_{i}
$$

By assuming that the transportation costs are usually charged on a per item. $\mathrm{km}$ basis, the following variables can be defined:

$T(x, y)$ : freight rate for shipments originating from $(x, y)$ (\$/item.km),

$k$ : a constant that depends on the distance metric and the shape of the service region in the neighborhood of $(x, y)$.

Each facility is assumed to be located at the center of its respective service region. Such assumption minimizes the average distance from the facility to each demand point inside the service region. Hence, annual transportation cost in region $R_{i}$ can be defined as:

$$
g\left(x_{i}, y_{i}, R_{i}\right)=\int_{R_{i}} T\left(x_{i}, y_{i}\right) \cdot d(x, y) \cdot D(x, y) d x d y
$$

The average distance between the facility and each demand point in its respective service region can be estimated by:

$\bar{d} \approx d(x, y) \approx k \cdot A_{i}^{1 / 2}$ 
Thus, we have:

$$
g\left(x_{i}, y_{i}, R_{i}\right) \approx T\left(x_{i}, y_{i}\right) \cdot k \cdot A_{i}^{1 / 2} \cdot D\left(x_{i}, y_{i}\right) \cdot A_{i}
$$

So, the total annual transportation cost is:

$$
\sum_{i} g\left(x_{i}, y_{i}, R_{i}\right)=\sum_{i} T\left(x_{i}, y_{i}\right) \cdot k \cdot D\left(x_{i}, y_{i}\right) \cdot A_{i}^{3 / 2}
$$

Accordingly, the facility location model is summarized as follows:

$$
\left\{\begin{array}{l}
\operatorname{Min} T C=\sum_{i} F\left(x_{i}, y_{i}\right)+O\left(x_{i}, y_{i}\right) \cdot D\left(x_{i}, y_{i}\right) \cdot A_{i}+\sum_{i} T\left(x_{i}, y_{i}\right) \cdot k \cdot D\left(x_{i}, y_{i}\right) \cdot A_{i}^{3 / 2} \\
\text { s.t. } \\
\bigcup_{i} R_{i}=M \\
R_{i} \cap R_{j}=\Phi ; \forall i \neq j \\
\left(x_{i}, y_{i}\right) \in R_{i} ; \forall i
\end{array}\right.
$$

The decision variables are the number $(n)$ and the location $\left(x_{i}, y_{i}\right)$ of facilities as well as the boundaries and areas of service regions ( $R_{i}$ 's and $A_{i}$ 's). All parameters are assumed to vary smoothly within a service region, yet the model allows large differences across areas likely to be served by different facilities.

Dasci and Verter [6] use some assumptions to solve problem (10). Their method ends to the result that given $(x, y)$ as a facility location point, the best area as its respective service region is:

$$
A^{*}=\left(\frac{2 F(x, y)}{T(x, y) \cdot k \cdot D(x, y)}\right)^{2 / 3}
$$

and the total annual cost corresponding to $A^{*}$ is estimated by:

$$
T C^{*}=\int_{M}\left(O(x, y) \cdot D(x, y)+(T(x, y) \cdot k \cdot D(x, y))^{2 / 3} \cdot F(x, y)^{1 / 3} \cdot \frac{3}{2^{2 / 3}}\right) d x d y
$$

Despite of the Dasci and Verter's excellent work [6], there are two cardinal problems to implement the initial model in practice. First, the model depends on four mathematical functions on a two-dimensional space; demand density, fixed cost, operational cost, and transportation cost. In real world problems, such functions are difficult, if not impossible, to be obtained by conventional regression models. For instance, consider a city as the service region on which we want to find a twodimensional mathematical function representing the demand density in each point. Obviously, such a function is extremely nonlinear having several extrema all over the service region. Thus, finding a mathematical function that fits on the sample data is not 
a trivial task. We are faced with the same problem about the three other functions.

The second problem arises in the selection of the points as facility locations. The initial model does not present any procedure to select the facility location points. The final solution of the initial model just states that given $(x, y)$ as a facility location point, the area of its respective service region is calculated by Eq. (11). So, the main questions are: which strategy should be used to select the first point, and how the selection of other facility location points should be continued?

\section{Fuzzy Modeling}

\subsection{Fuzzy Rule Base}

Since fuzzy sets theory was first introduced by Zadeh in 1965 [26], it has impressed a wide variety of disciplines. Among the applications of fuzzy sets theory, Fuzzy Rule Base (FRB) is a popular technique in which the relation between inputs and outputs of a system is made in the form of fuzzy if-then rules rather than a pure mathematical function. Each rule in a Takagi-Sugeno FRB (TSFRB), a especial type of FRBs, consists of two main parts: antecedent (if part) and consequent (then part) as follows: If $X_{1}$ is $\mu_{i 1}\left(x_{1}\right)$ and $X_{2}$ is $\mu_{i 2}\left(x_{2}\right)$ and $\ldots$ and $X_{m}$ is $\mu_{i m}\left(x_{m}\right)$ Then $\hat{y}_{i}=a_{i 0}+\sum_{j=1}^{m} a_{i j} \cdot x_{j}$

TSFRBs can be viewed as an expansion of piecewise linear partitioning in which fuzzy transitions between the linear functions allow for the modeling of complex nonlinear systems with a good global accuracy. Given a TSFRB and the input vector, $X=\left(x_{1}, x_{2}, \ldots, x_{m}\right)$, the inference procedure is as follows:

1) fuzzification: calculate the degree of membership of the $j$ th element of the input vector in its corresponding fuzzy number in the $i$ th rule:

$$
\mu_{i j}\left(x_{j}\right) ; i=1,2, \ldots, c ; j=1,2, \ldots, m
$$

where, $c$ is the number of rules.

2) degree of matching: calculate the degree of matching of the input vector, $X$, with the $i$ th rule:

$$
\mu_{i}(X)=\prod_{j=1}^{m} \mu_{i j}\left(x_{j}\right) ; i=1,2, \ldots, c
$$


3) output of each rule: calculate the output of each rule using input vector and consequent of that rule:

$$
\hat{y}_{i}(X)=a_{i 0}+\sum_{j=1}^{m} a_{i j} \cdot x_{j}
$$

4) aggregation: calculate the weighted average of the outputs:

$$
\hat{y}(X)=\frac{\sum_{i=1}^{c} \mu_{i}(X) \cdot \hat{y}_{i}(X)}{\sum_{i=1}^{c} \mu_{i}(X)}
$$

$\hat{y}(X)$ is the final output of the FRB.

\subsection{Clustering}

The process of extracting a FRB from input-output data of the system is an interesting and promising research area called fuzzy modeling. Fuzzy modeling is a powerful technique to estimate nonlinear functions in the form of a FRB. Many methods have been proposed by different authors in this domain $[4,13,15,22,23]$.

Fuzzy modeling methods usually comprise two main phases: 1) structure identification (rough tuning), and 2) parameter identification (fine tuning). Structure identification is mostly associated with partitioning of the input-output space, whereas parameter identification concerns to estimating parameters of the Membership Functions (MFs) and coefficients of the linear functions. In other words, the purpose of structure identification is to construct an initial fuzzy model to describe the inherent structure of the given input-output data, while the procedure of parameter identification is applied to obtain a more precise fuzzy model via determination of the most appropriate MFs and coefficients of linear functions.

Clustering algorithms are the most popular tools for structure identification by which we deal with partitioning of the input-output space and assigning MFs to the partitions. Sadrabadi and Zarandi [21] propose an algorithm to classify input-output points into two categories: the points located in the linear parts and the point located in the extrema. This is preparation of the data for fuzzy clustering, and a special clustering algorithm is appropriate to be implemented on each category. In our proposed method to fuzzy modeling we use subtractive clustering for structure identification. Subtractive clustering was introduced by Chiu [5] in which each data point is considered as a 
potential cluster center. Such a potentiality is calculated for each data point, $X_{k}$, based on the density of other surrounding data points. Each time a cluster center is obtained, the data points in the vicinity of the previous cluster center are removed in order to facilitate the emergence of the new cluster center. Subtractive clustering algorithm is as follows:

\section{Subtractive Clustering Algorithm}

\section{Begin:}

Step 1. set $i=1$ and calculate the potentiality of each data point as a cluster center in the first iteration by:

$$
D_{k}(1)=\sum_{l=1}^{n} \exp \left(-\frac{\left\|X_{k}-X_{l}\right\|}{\left(r_{1} / 2\right)^{2}}\right) ; k=1,2, \ldots, n
$$

where, $r_{1}$ is a positive constant defining the neighborhood range of the cluster or simply the radius of hypersphere cluster in data space.

Step 2. select the data point with the highest potentiality in the first iteration as the first cluster center. In other words, select $X_{c_{1}}$ as the first cluster center, such that:

$$
D_{c_{1}}=\max \left\{D_{k}(1) ; k=1,2, \ldots, n\right\}
$$

Step 3. set $i=2$ and calculate the revised potentiality of the remaining data points in the second iteration by:

$$
D_{k}(2)=D_{k}(1)-D_{c_{1}} \cdot \exp \left(-\frac{\left\|X_{k}-X_{c_{1}}\right\|}{\left(r_{2} / 2\right)^{2}}\right) ; k=1,2, \ldots, n ; k \neq c_{1}
$$

where,

$$
r_{2}=\eta r_{1}
$$

and $D_{c_{1}}$ is the potentiality of the first cluster center in the previous iteration. The positive constant, $r_{2}$, defines the efficient subtractive range somewhat greater than $r_{1}$ which helps avoiding closely spaced cluster centers; so, squash factor, $\eta$, is a positive constant greater than 1 .

Step 4. select $X_{c_{2}}$ as the second cluster center such that:

$$
D_{c_{2}}=\max \left\{D_{k}(2) ; k=1,2, \ldots, n ; k \neq c_{1}\right\}
$$

Step 5. set $i=i+1$ and calculate the revised potentialities of the points in the $i$ th iteration by:

$$
D_{k}(i)=D_{k}(i-1)-D_{c_{i-1}} \cdot \exp \left(-\frac{\left\|X_{k}-X_{c_{i-1}}\right\|}{\left(r_{i} / 2\right)^{2}}\right) ; k=1,2, \ldots, n ; k \neq c_{1}, c_{2}, \ldots, c_{i-1}
$$


where,

$$
r_{i}=\eta r_{i-1}
$$

Step 6. consider $X_{c_{i}}$ as the only candidate for the $i$ th cluster center such that:

$$
D_{c_{i}}=\max \left\{D_{k}(\mathrm{i}) ; k=1,2, \ldots, n ; k \neq c_{1}, c_{2}, \ldots, c_{i-1}\right\}
$$

Step 7. if $D_{c_{i}}<\underline{\varepsilon}$ the algorithm is terminated without selecting the $i$ th cluster center, otherwise go to step 8. $\underline{\varepsilon}$ is a rejection threshold.

Step 8. if $D_{c_{i}}>\bar{\varepsilon}$ select $X_{c_{i}}$ as the $i$ th cluster center and go to step 5, otherwise go to step 9. $\overline{\mathcal{E}}$ is an acceptance threshold.

Step 9. if $\underline{\varepsilon} \leq D_{c_{i}} \leq \bar{\varepsilon}$ and inequality:

$\frac{d_{i}}{r_{1}}+\frac{D_{c_{i}}}{D_{c_{1}}} \geq 1$

where,

$$
d_{i}=\min \left\{\left\|X_{c_{i}}-X_{c_{l}}\right\| ; l=1,2, \ldots, i-1\right\}
$$

holds, select $X_{c_{i}}$ as the $i$ th cluster center and go to step 5, otherwise terminate the algorithm without selecting the $i$ th cluster center. $\|$.$\| is the Euclidean norm.$

\section{End.}

As mentioned in the algorithm, subtractive clustering has four parameters, namely, acceptance threshold $\bar{\varepsilon}$, reject threshold $\underline{\varepsilon}$, cluster radius $r_{1}$, and squash factor $\eta$. These parameters have influence on the number of rules and error performance measures. Large values of $\bar{\varepsilon}$ and $\underline{\varepsilon}$ will result in small number of rules. Conversely, small values of $\bar{\varepsilon}$ and $\underline{\varepsilon}$ will increase the number of rules. A large value of $r_{1}$ generally results in fewer clusters that leads to a coarse model, whereas a small value of $r_{1}$ produces excessive number of rules that may result in an overfitted system. The suggested values for $\eta$ and $r_{1}$ are $1.25 \leq \eta \leq 1.5$ and $0.2 \leq r_{1} \leq 0.5[5,17]$. In this paper, $r_{1}=0.4, \eta=1.25, \bar{\varepsilon}=0.5, \underline{\varepsilon}=0.15$ are considered.

It should be noted that a method for scaleless of data must be applied before data clustering, in that different dimensions of the data can be of different scales. The data in this paper are interval data which can be scalelessed as follows [9]: 
$z_{k j}=\frac{x_{k j}-m_{j}^{\prime}}{s_{j}^{\prime}} ; k=1,2, \ldots, n ; j=1,2, \ldots, m$

where,

$$
\begin{aligned}
& m_{j}^{\prime}=\frac{\sum_{k=1}^{n} x_{k j}}{n} ; j=1,2, \ldots, m \\
& s_{j}^{\prime}=\frac{\sum_{k=1}^{n}\left|x_{k j}-m_{j}\right|}{n} ; j=1,2, \ldots, m
\end{aligned}
$$

After clustering, the cluster centers are returned to the initial scale using:

$$
x_{k j}=m_{j}^{\prime}+s_{j}^{\prime} \cdot z_{k j} ; k=1,2, \ldots, n ; j=1,2, \ldots, m
$$

\subsection{The Proposed Method to Fuzzy Modeling}

In this section, a fuzzy modeling method is proposed in which subtractive clustering is used for structure identification. Moreover, parameters of the antecedents and the consequents are identified by some heuristics and an optimization problem, respectively. Suppose sample input-output data of a system are given as:

$$
\left(X_{k}, y_{k}\right)=\left(x_{k 1}, x_{k 2}, \ldots, x_{k m}, y_{k}\right) ; k=1,2, \ldots, n
$$

based on which we are going to extract a TSFRB in order to make the relation between input vector and output of the system. In the proposed method, Gaussian MFs are considered to guarantee that the whole of the input space is covered. Gaussian MFs are represented as:

$$
\mu(x)=\exp \left(-0.5\left(\frac{x-m}{s}\right)^{2}\right)
$$

Generally, a FRB consists of $p$ rules in a $m$-dimensional input space; so, MF of the $j$ th variable in the antecedent of the $i$ th rule can be presented as:

$$
\mu_{i j}\left(x_{j}\right)=\exp \left(-0.5\left(\frac{x_{j}-m_{i j}}{s_{i j}}\right)^{2}\right)
$$

Furthermore, the linear function in the consequent of the $i$ th rule can be presented as:

$$
\hat{y}_{i}(X)=a_{i 0}+\sum_{j=1}^{m} a_{i j} \cdot x_{j}
$$


Therefore, the parameters of the FRB are the antecedent parameters, $m_{i j}, s_{i j} ; i=1,2, \ldots, p ; j=1,2, \ldots, m$ and the consequent parameters, $a_{i 0}, a_{i j} ; i=1,2, \ldots, p ; j=1,2, \ldots, m$.

Obviously, structure of the FRB must be specified before parameter identification, i.e., the number of rules and rough partitioning of the input space must be specified.

\section{Structure Identification}

For structure identification, subtractive clustering on the input-output space is applied. This leads to identifying the number of clusters, $p$, as well as the center of each cluster:

$$
\left(m_{i 1}, m_{i 2}, \ldots, m_{i m}\right)=C_{i}=X_{c_{i}}=\left(x_{c_{i} 1}, x_{c_{i} 2}, \ldots, x_{c_{i} m}\right) ; i=1,2, \ldots, p
$$

The weighted variance of the $j$ th MF in the $i$ th cluster is estimated by:

$$
s_{i j}^{2}=\frac{\sum_{k=1}^{n} w_{k i j} \cdot\left(x_{k j}-m_{i j}\right)^{2}}{\sum_{k=1}^{n} w_{k i j}} ; i=1,2, \ldots, p ; j=1,2, \ldots, m
$$

where,

$$
w_{k i j}=\frac{\exp \left(-\beta .\left|x_{k j}-m_{i j}\right|\right)}{\sum_{i=1}^{p} \exp \left(-\beta .\left|x_{k j}-m_{i j}\right|\right)} k=1,2, \ldots, n ; i=1,2, \ldots, p ; j=1,2, \ldots, m
$$

and $\beta$ is an arbitrary coefficient. The bigger value of $\beta$ implies the more stress on the data near the cluster center to determine the variance of its respective MF. In other words, the bigger value of $\beta$ leads to MFs with less measures of fuzziness. In this paper, $\beta=0.25$ is considered for its satisfactory results in several numerical examples; it leads to more accurate FRBs.

\section{Parameter Identification}

An optimization problem is used to calculate the consequent parameters of the FRB where sum of squared errors is minimized. By considering the MF of the $j$ th variable in the $i$ th rule as $\mu_{i j}\left(x_{j}\right)$, the degree of matching of input vector $X$ in the $i$ th rule is calculated as:

$$
\mu_{i}(X)=\prod_{j=1}^{m} \mu_{i j}\left(x_{j}\right) ; i=1,2, \ldots, p
$$

Moreover, the output of each rule is calculated by: 


$$
\hat{y}_{i}(X)=a_{i 0}+\sum_{j=1}^{m} a_{i j} \cdot x_{j}
$$

Eventually, the final output of the input vector $X$ is:

$$
\hat{y}(X)=\frac{\sum_{i=1}^{p} \mu_{i}(X) \cdot \hat{y}_{i}(X)}{\sum_{i=1}^{p} \mu_{i}(X)}
$$

$\hat{y}(X)$ is the estimated output for the input $X$ by FRB. Given the real value of the output, $y(X)$, the optimization problem, in which the decision variables are coefficients of the linear functions in consequents, is as follow:

$$
\left\{\begin{array}{l}
\operatorname{Min} \sum_{k=1}^{n}\left(y\left(X^{k}\right)-\hat{y}\left(X^{k}\right)\right)^{2} \\
\hat{y}\left(X^{k}\right)=\frac{\sum_{i=1}^{p} \mu_{i}\left(X^{k}\right) \cdot \hat{y}_{i}\left(X^{k}\right)}{\sum_{i=1}^{p} \mu_{i}\left(X^{k}\right)} ; k=1,2, \ldots, n \\
\hat{y}_{i}\left(X^{k}\right)=a_{i 0}+\sum_{j=1}^{m} a_{i j} x_{j}^{k} ; i=1,2, \ldots, p ; k=1,2, \ldots, n \\
\mu_{i}\left(X_{k}\right)=\prod_{j=1}^{m} \mu_{i j}\left(x_{j}\right) ; i=1,2, \ldots, p ; k=1,2, \ldots, n \\
\mu_{i j}\left(x_{k j}\right)=\exp \left(-0.5\left(\frac{x_{k j}-m_{i j}}{s_{i j}}\right)^{2}\right) ; i=1,2, \ldots, p ; j=1,2, \ldots, m ; k=1,2, \ldots, n
\end{array}\right.
$$

By solving the above problem, parameter identification of the FRB is completed.

\section{Development of the Initial Model}

\subsection{Estimating the Required Functions via Fuzzy Modeling}

In order to utilize fuzzy modeling to resolve the first mentioned problem in section 2, we first specify the service region and cover it by small cells as indicated in Fig. 2.

Fig. 2. Covering the service region by small cells.

This strategy can be regarded as a bridge between continuous and discrete facility location models and is applicable by any arbitrary precision; the smaller cells, the more precise model, but more computational effort is needed.

Each cell encompasses a certain region with area $s$ and is specified by an ordered pair $(c, r)$ where $c$ and $r$ indicate the cell's column and row, respectively. Here, the lower 
left corner is considered as the center of coordination axes. Then, $n$ cells are selected randomly and their respective values of functions $D, F, O$, and $T$ are determined as their average values in the corresponding cell. Accordingly, the sample data are in hand as indicated in Table I.

Table I Sample data of functions.

The input-output data in Table I is used to extract four distinct FRBs. In the all FRBs $(c, r)$ are input variables and $D, F, O$, and $T$ are output variables of the four FRBs, respectively. After extracting the four above mentioned FRBs and given $(c, r)$, the average value of each function in its respective cell can be estimated. So, the first problem of the initial model can be resolved via fuzzy modeling.

\subsection{Implementation of the Modified Model via Simulation in a Discrete Area}

To select the cells to locate the facilities, a strategy must be specified. Let $c_{\text {max }}$ and $r_{\max }$ be the number of columns and rows of a hypothetical rectangle encompassing the entire service region, respectively. So, the total number of the cells of the rectangle is:

$$
q=c_{\text {max }} \cdot r_{\max }
$$

Obviously, some of the cells are outside the service region inadmissible to locate facilities in them. Moreover, it is likely to exclude some cells inside the service region inasmuch as the facility locations are not permitted to be there. For example, an airport, a park, or an extensive residence area even though might have demand but are not permitted to locate the facilities in them. Let the cell located in the lower left corner of the hypothetical rectangle indicates $\mathrm{Cell}_{1}$ and the cell located in the upper right corner of the hypothetical rectangle indicates $\mathrm{Cell}_{q}$. There is a unique relation between the cell number and its row and column as follows:

$$
\begin{aligned}
& \text { Cell }_{k}=c_{\text {max }} \cdot(r-1)+c \\
& c=\text { Cell }_{k}-c_{\text {max }} \cdot\left[\text { Cell }_{k} / c_{\text {max }}\right] \\
& r=\left[\text { Cell }_{k} / c_{\text {max }}\right]+1
\end{aligned}
$$

In order to determine the location of the first facility, we generate a random integer $1 \leq k \leq q$. If Cell $_{k}$ is an inadmissible cell, we generate another random integer, otherwise its respective row and column, $(c, r)$, is characterized by Eqs. (43)-(44). In 
such a case, $(c, r)$ are considered as input values of the four FRBs, and the output value from each FRB is calculated based on the fuzzy inference, i.e., $D(c, r), F(c, r), O(c, r)$ , and $T(c, r)$. In the next step, the area that should be served by $\mathrm{Cell}_{k}$ is calculated by:

$$
A(c, r)=\left(\frac{2 F(c, r)}{T(c, r) \cdot k \cdot D(c, r)}\right)^{2 / 3}
$$

Then, coordination of the center of the cell $(c, r)$ is determined using:

$$
\begin{aligned}
& x=\sqrt{s} .(c-0.5) \\
& y=\sqrt{s} .(r-0.5)
\end{aligned}
$$

where, $s$ is the area of each cell.

The number of cells that must be served by the first facility is calculated as,

$$
n(c, r)=[A(c, r) / s]+1
$$

The first facility is located on the point $(x, y)$ and its respective cell is considered as the first covered cell. Then, its neighbor cells are annularly covered until the number of cells meets $n(c, r)$. When an inadmissible cell is encountered it is skipped and the process proceeds to cover other cells. Fig. 3(a) shows a sample solution with $n(6,12)=28$ for the first cell.

Fig. 3. An iteration of the simulation process: (a) selecting the first location, (b) selecting the second location, (c) all possible locations, and (d) allocating all cells to the suitable facility.

In this paper, the facilities are supposed to serve a square-shape area around themselves. Moreover, rectangular metrics are used. Thus, average distance from the facility located in the center of the square to any point inside it is:

$$
\bar{d}=\frac{\int_{-a / 2}^{a / 2}(|x|+|y|) d x d y}{\int_{-a / 2}^{a / 2} d x d y}=\frac{0.5 a^{3}}{a^{2}}=0.5 \sqrt{a^{2}}=0.5 \sqrt{A}
$$

Therefore, constant $k=0.5$ is assigned in Eq. (45).

The all cells assigned to the first facility are considered as the set $S_{1}$. Then, all Cell $_{k} \in S_{1}$ are added to the set of inadmissible cells. The process of locating the second facility is similar to the first one. Fig. 3(b) indicates the sample solution in which the first two facilities are located.

After locating some facilities, an admissible cell might be selected to locate the next 
facility, yet the new cell is in a narrow bar between two previously served areas. In such cases, the new facility can not serve a square area. Hence, it is better to select another cell, in that it is an undesirable situation. For the sake of simplicity in the following relations, suppose:

$k=c_{\max } \cdot(r-1)+c$

Such cases can be controlled by:

$$
\begin{aligned}
& \left|c_{k}-c\right|>\frac{\sqrt{A_{k}}+\sqrt{A}}{2 \sqrt{s}} \\
& \left|r_{k}-r\right|>\frac{\sqrt{A_{k}}+\sqrt{A}}{2 \sqrt{s}}
\end{aligned}
$$

If at least one of the Eqs. (51)-(52) holds for all k's (for all located facilities before the new one), the selected cell is considered as location of the new facility, otherwise another integer number is generated. Generation of random integers is continued till no cell is remaining able to satisfy Eq. (51) or (52). Fig. 3(c) shows the situation in which Eqs. (51)-(52) are no longer satisfied by any point. In this case, there are some cells that are not served by any facility, and yet no facility can be located on the service region, as they do not satisfy Eqs. (51)-(52). Since all over the service region must be served, the remaining cells are assigned to the located facilities via an allocation function. Assigning the remaining $\mathrm{Cell}_{l}$ to the located facility $k$ imposes cost $h_{k l}$ on the facility consisting of the operational cost and the transportation cost as:

$$
h_{k l}=O_{k} \cdot D_{l} \cdot s+\left(\frac{T_{k}+T_{l}}{2}\right) \cdot D_{l} \cdot s^{3 / 2} \cdot\left(\left|c_{k}-c_{l}\right|+\left|r_{k}-r_{l}\right|\right)
$$

Cell $l$ is assigned to the facility, the respective $h_{k l}$ of which is minimum in comparison to the other facilities. Similarly, other remaining cells are assigned to the proper facility based on the amounts of $h_{k l}$ 's.

Solving several numerical examples has shown that it is better to calculate $h_{k l}$ not only for the remaining cells, but also for the served cells. Hence, $h_{k l}$ is calculated for all $k$ 's and all cells. This might lead to eliminating some located facilities and so reduction in their number; when all the cells around a facility are allocated to another facility. Fig. 3(d) shows the final solution after the allocation of cells to suitable facilities. After allocation, the total annual cost caused by the $k$ th facility can be calculated by: 


$$
\begin{aligned}
& T C_{k}=F_{k}+O_{k} \cdot D_{k} \cdot s+T_{k} \cdot D_{k} \cdot s^{3 / 2} \cdot k+\sum_{l \in S_{k}} O_{k} \cdot D_{l} \cdot s \\
& +\sum_{l \in S_{k}}\left(\frac{T_{k}+T_{l}}{2}\right) \cdot D_{l} \cdot s^{3 / 2} \cdot\left(\left|c_{k}-c_{l}\right|+\left|r_{k}-r_{l}\right|\right)
\end{aligned}
$$

and the total annual cost caused by all the facilities is,

$$
T C=\sum_{k=1}^{m} T C_{k}
$$

$T C$ shows the total annual cost of the first iteration of the simulation process. The simulation process is carried out several times, and the plan with the lowest $T C$ is selected as the final one.

Implementation of the modified model via computer programming by Visual Basic 6 has been successfully carried out. In this program, the number of iterations is specified arbitrary, and plan generation is carried out automatically. Finally, the best plan is shown along with its respective $T C$.

\section{Numerical Example}

This section provides a numerical example to demonstrate the proposed fuzzy modeling method and also implementation of this method along with the proposed heuristic relations to develop the initial facility location model. As discussed in the previous sections, the first step to fuzzy modeling is gathering numerical input-output data of the system under studied. Here, the system is the region $M$ in which the cell coordination, $(c, r)$, is the input and $D, F, O$, and $T$ are the outputs. Since transportation cost, $T$, is usually constant, we consider $T(c, r)=0.0008$. Table II shows 106 cells in the region $M$ for which the other required numerical input-output data are gathered.

Table II Numerical input-output data for region $M$.

Since the variables are from different scales, they should be scalelessed before subtractive clustering is applied; so, $m^{\prime}$ and $s^{\prime}$ are calculated. The values of $m^{\prime}$ and $s^{\prime}$ for each dimension are presented in Table III. Accordingly, the original data are scalelessed based upon Table II and Eqs. (27)-(29).

Table III Mean and standard deviation of each dimension.

Now, a distinct FRB should be constructed to obtain each output $D, F$, and $O$. Obviously, $(c, r)$ is the input for all FRBs. Therefore, subtractive clustering is applied 
on the scalelessed data $\left(c_{\text {Scalelessed }}, r_{\text {Scalelessed }}, D_{\text {Scalelessed }}\right)$ to obtain the number of clusters and to specify the center of each cluster in the first FRB. Table IV shows the results.

Table IV Center of each cluster in both the scalelessed dimensions and the rescalled dimensions in the FRB with the output $D$.

Similarly, Tables V-VI show the results of implementation of subtractive clustering to construct the second and the third FRB.

Table V Center of each cluster in both the scalelessed dimensions and the rescalled dimensions in the FRB with the output $F$.

Table VI Center of each cluster in both the scalelessed dimensions and the rescalled dimensions in the FRB with the output $O$.

Now, the values $s_{c}$ and $s_{r}$ can be calculated using Eqs. (36)-(37). Hence, parameter identification of the antecedents is completed. Then, coefficients of the linear functions in the consequents are determined based on the parameters of the antecedents and using the optimization problem (40). The results for the first FRB are listed in Table VII.

Table VII Parameters of the FRB with the output $D$.

According to the above table, the $i$ th rule can be written as,

$$
\text { Rule }_{i} \text { : If c is } \mu_{i}(c)=\exp \left(-0.5\left(\frac{c-m_{c}(i)}{s_{c}(i)}\right)^{2}\right) \text { and } r \text { is } \mu_{i}(r)=\exp \left(-0.5\left(\frac{r-m_{r}(i)}{s_{r}(i)}\right)^{2}\right)
$$

Then $D=a(i) c+b(i) r+c(i)$

The first rule, for example, is,

$$
\begin{aligned}
& \text { Rule }_{1} \text { : If } c \text { is } \mu_{1}(c)=\exp \left(-0.5\left(\frac{c-29.998}{6.196}\right)^{2}\right) \text { and } r \text { is } \mu_{1}(r)=\exp \left(-0.5\left(\frac{r-24.996}{5.652}\right)^{2}\right) \\
& \text { Then } D=-2.905 c-0.204 r+565.297
\end{aligned}
$$

In order to evaluate the efficiency of each FRB, Mean of Squared Errors (MSE) and Mean of Relative Absolute Errors (MRAE) are reliable criteria which are defined as:

$$
\begin{aligned}
& M S E=\frac{\sum_{k=1}^{n}\left(\text { Output }_{k}^{R}-\text { Output }_{k}^{M}\right)^{2}}{n} \\
& M R A E=\frac{\sum_{k=1}^{n} \frac{\mid \text { Output }_{k}^{R}-\text { Output }_{k}^{M} \mid}{\text { Output }_{k}^{R}}}{n}
\end{aligned}
$$


where, Output $_{k}^{R}$ and $O u t p u t_{k}^{M}$ are the real output and the model's output, respectively, for the $k$ th data. For the FRB presented in Table VI we have MSE=983.309 that with regard to the values of $D$ is an acceptable error. Fig. 4 shows the first FRB with the output $D$ in a 3 -dimensional space. We also have $M R A E=0.041$ for this FRB.

Fig. 4. The FRB with the output $D$

Similarly, Tables VIII-IX present the parameters of the second and the third FRB with the output $F$ and $O$, respectively.

Table VIII Parameters of the FRB with the output $F$.

Table IX Parameters of the FRB with the output $O$.

The $M S E$ for the second and the third FRB are $M S E=400.729$ and $M S E=0.000009$, respectively. Again, regarding to the values of $F$ and $O$, the obtained MSEs show acceptable FRBs. The values $M R A E=0.003$ and $M R A E=0.012$ are also obtained for the second and the third FRB, respectively. Figs. 5-6 show the FRBs associated with $F$ and $O$ in a 3 -dimensional space.

Fig. 5. The FRB with the output $F$

Fig. 6. The FRB with the output $O$

Now, the required FRBs with good accuracy are in hand. We cover region $M$ presented in Fig. 7 by $c \times r=90 \times 60=5400$ cells.

Fig. 7. Covering the region $M$ by cells

Suppose that each cell has the area $s=4 \mathrm{~km}^{2}$. Also, $k=0.5$ is considered as the shape factor, in that rectangular metrics are considered as the distance criterion.

Suppose that the first random selected cell is $\left(c_{1}, r_{1}\right)=(24,28)$. By using the FRBs and the other required relations, we have $n\left(c_{1}, r_{1}\right)=n(24,28)=410$. Therefore, the first facility is located in the cell $(24,28)$ and should cover 410 cells. This is presented in Fig. 8 with blue color. Suppose that the next three cells are $\left(c_{2}, r_{2}\right)=(51,13)$ with $n\left(c_{2}, r_{2}\right)=612,\left(c_{3}, r_{3}\right)=(49,40)$ with $n\left(c_{3}, r_{3}\right)=348$, and $\left(c_{4}, r_{4}\right)=(72,38)$ with $n\left(c_{4}, r_{4}\right)=329$, represented in Fig. 8 with red, green, and yellow colors, respectively.

Fig. 8. The random selected cells and their respective service regions.

After locating the four presented facilities, there are no other cells which can satisfy conditions (51)-(52). Hence, the remaining cells are assigned to the most suitable facility, according to Eq. (53). Fig. 9 shows the result of such assignment. 
Fig. 9. Assigning the other cells to the located facilities.

The presented solution in Fig. 9 is not the best assignment, in that some cells can be allocated to a different facility with lower cost. So, all cells in the region $M$ are allocated to the most suitable located facility. Fig. 10 shows the result of such allocation.

Fig. 10. Allocating all cells to the most suitable located facility.

Fig. 10 is the final solution for the first iteration of the simulation process with, $T C_{1}=230098, T C_{2}=265555, T C_{3}=342108$, and $T C_{4}=250582$. The total annual cost for the first iteration is $T C=1088343$ which is considered as the criterion for evaluation of this solution. Other simulation iterations are conducted by selecting new random cells and calculating the final $T C$. The best solution is one with the lowest $T C$.

\section{Conclusion and Future Works}

In this paper, a fuzzy modeling method has been proposed to develop a continuous facility location model in the literature. Four distinct FRBs have been extracted based upon sample input-output data. Subtractive clustering, some heuristics, and an optimization problem have been used to identify the FRB. Moreover, some heuristic relations have been proposed to implement the modified model via simulation in an arbitrary discrete space and to evaluate the simulation plans. Implementation of the modified model has been carried out successfully by computer programming in Visual Basic 6. A complete numerical example has been presented in the paper to demonstrate implementation of the proposed method to fuzzy modeling and development of the initial facility location model via the proposed heuristic relations. Future works can be associated with elimination of some assumptions in the initial model; the assumption of smooth transition of the functions, for example. It likely changes some relations in the initial model and so the other following relations.

\section{References}

[1] Benjaafar S., Heragu S.S., and Irani S.A. Next generation factory layouts: research challenges and recent progress. Interfaces 2002; 32(6); 58-76.

[2] Chen C.T. A fuzzy approach to select the location of the distribution center. Fuzzy Sets and Systems 2001; 118; 65-73.

[3] Chen S.M. Fuzzy group decision-making for evaluating the rate of aggregative risk in software development. Fuzzy Sets and Systems 2001; 118; 75-88. 
[4] Chen M.Y. and Linkens D.A. Rule-base self-generation and simplification for data-driven fuzzy models. Fuzzy Sets and Systems 2004; 142; 243-265.

[5] Chiu S. Fuzzy model identification based on cluster estimation. Journal of Intelligent Fuzzy Systems 1994; 2(3); 267-278.

[6] Dasci A. and Verter V. A continuous model for production-distribution system design. European Journal of Operational Research 2001; 129; 287-298.

[7] Diaz-Banez J.M., Mesa J.A., and Schobel A. Continuous location of dimensional structures. European Journal of Operational Research 2004; 152; 22-44.

[8] Hamacher H.W. and Nickel S. Classification of location models. Location Science 1998; 6; 229-242.

[9] Han J. and Kamber M. Data mining: concepts and techniques. Boston: Morgan Kaufmann Publishers; 2006. p. 386-397.

[10] Kahraman C., Ruan D., and Dogan I. Fuzzy group decision-making for facility location selection. Information Sciences 2003; 157; 135-153.

[11] Keskin B.B. and Uster H. Meta-heuristic approaches with memory and evolution for a multi-product production/distribution system design problem. European Journal of Operational Research 2007; 182(2); 663-682.

[12] Khilwani N., Shankar R., and Tiwari M.K. Facility layout problem: an approach based on a group decision-making system and psychoclonal algorithm. International Journal of Production Research 2008; 46(4); 895-927.

[13] Kim E., Park Min., Ji S., and Park Mig. A new approach to fuzzy modeling. IEEE Transactions on Fuzzy Systems 1997; 5(3); 328-337.

[14] Klose A. and Drexl A. Facility location models for distribution system design. European Journal of Operational Research 2005; 162; 4-29.

[15] Kosko B. Fuzzy engineering. New Jersey: Prentice Hall International; 1997. p. 139-173.

[16] Kuo R.J., Chi S.C., and Kao S.S. A decision support system for selecting convenience store location through integration of fuzzy AHP and artificial neural network. Computers in Industry 2002; 47(2); 199-214.

[17] MATLAB 7.1 Help.

[18] Moreno Perez J.A., Moreno Vega J.M., and Verdegay J.L. Fuzzy location problems on networks. Fuzzy Sets and Systems 2004; 142; 393-405.

[19] Plastria F. Static competitive facility location: an overview of optimization approaches. European Journal of Operational Research 2001; 129; 461-470. 
[20] ReVelle C.S. and Eiselt H.A. Location analysis: a synthesis and survey. European Journal of Operational Research 2005; 165; 1-19.

[21] Rezaei Sadrabadi M. and Fazel Zarandi M.H. Identification of the linear parts of nonlinear systems for fuzzy modeling. Applied Soft Computing 2010; Article in press.

[22] Sugeno M. and Yasukawa T. A fuzzy-logic-based approach to qualitative modeling. IEEE Transactions on Fuzzy Systems 1993; 1(1); 7-31.

[23] Takagi T. and Sugeno M. Fuzzy identification of systems and its applications to modeling and control. IEEE Transactions on Systems, Man, and Cybernetics 1985; 15(1); 116-132.

[24] Tzeng G.H. and Chen Y.W. Optimal location of airport fire stations: a fuzzy multiobjective programming and revised genetic algorithm approach. Transportation Planning and Technology 1999; 23(1); 37-55.

[25] Wen M. and Kang R. Some optimal models for facility location-allocation problem with random fuzzy demands. Applied Soft Computing 2010; Article in press.

[26] Zadeh L.A. Fuzzy sets. Information and Control 1965; 8; 338-353. 


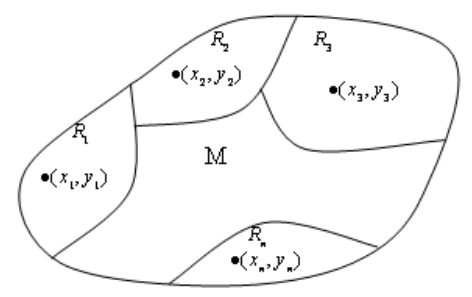

Fig. 1. A sample solution of the initial model.

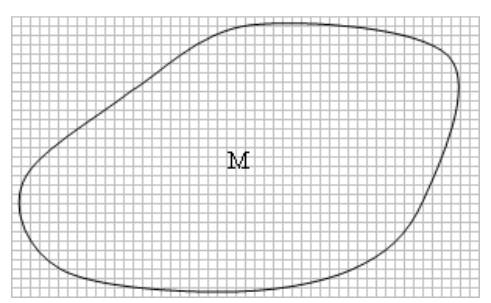

Fig. 2. Covering the service region by small cells.

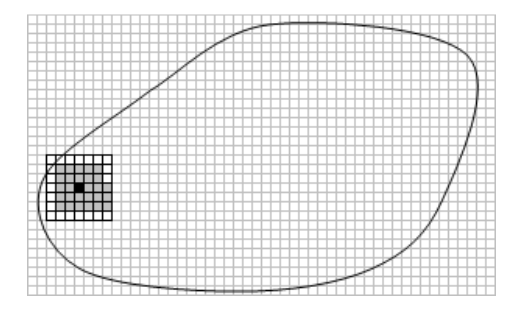

(a)

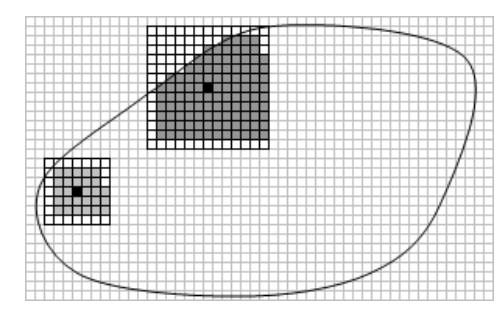

(b)

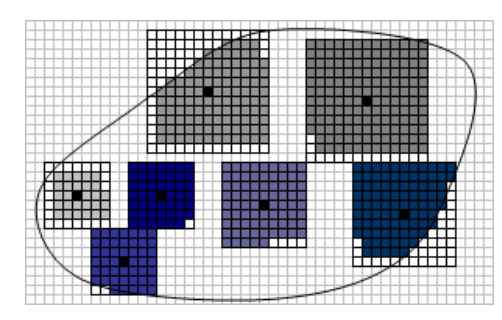

(c)

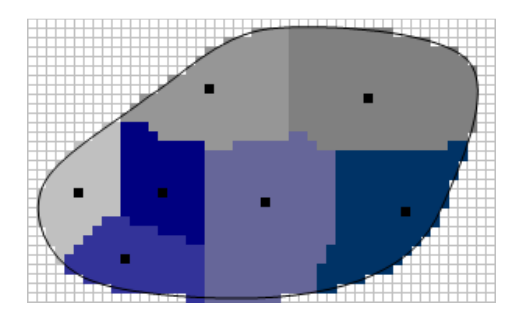

(d) 
Fig. 3. An iteration of the simulation process: (a) selecting the first location, (b) selecting the second location, (c) all possible locations, and (d) allocating all cells to the suitable facility.

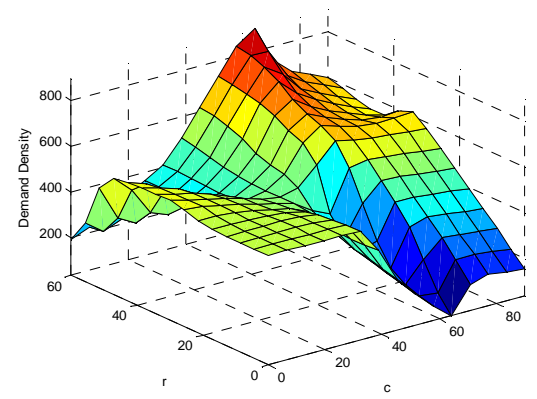

Fig. 4. The FRB with the output $D$

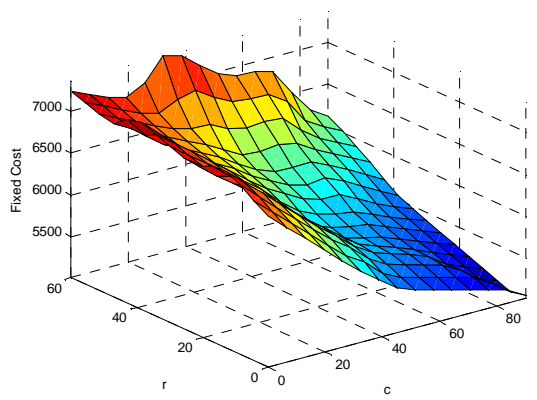

Fig. 5. The FRB with the output $F$

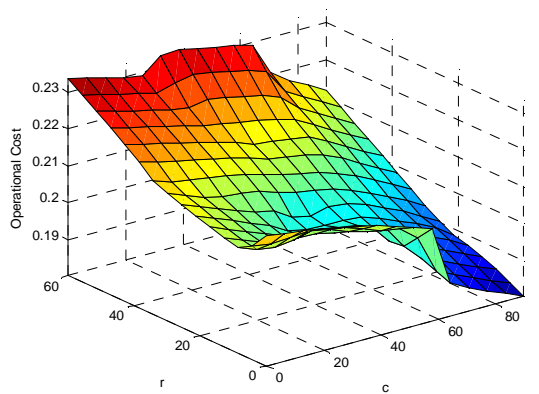

Fig. 6. The FRB with the output $O$ 


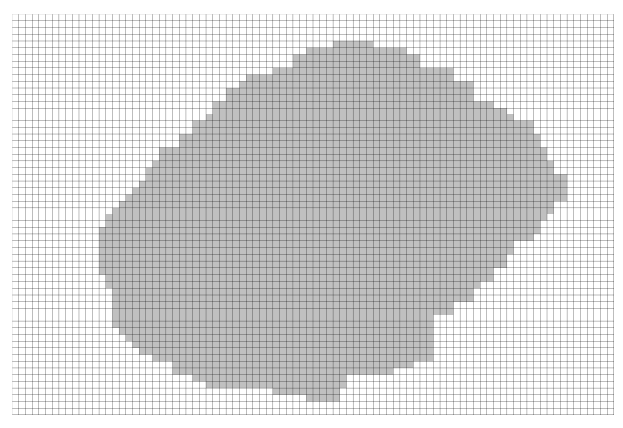

Fig. 7. Covering the region $M$ by cells

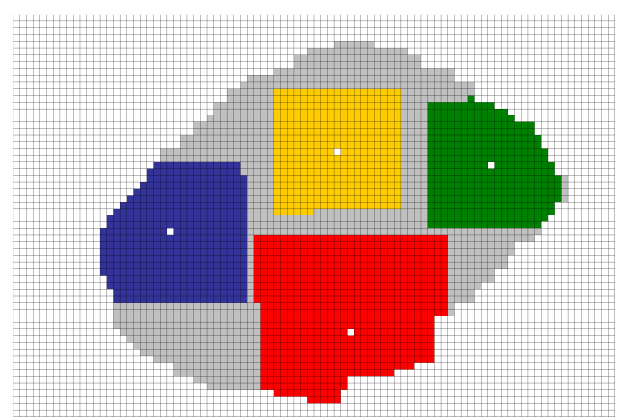

Fig. 8. The random selected cells and their respective service regions.

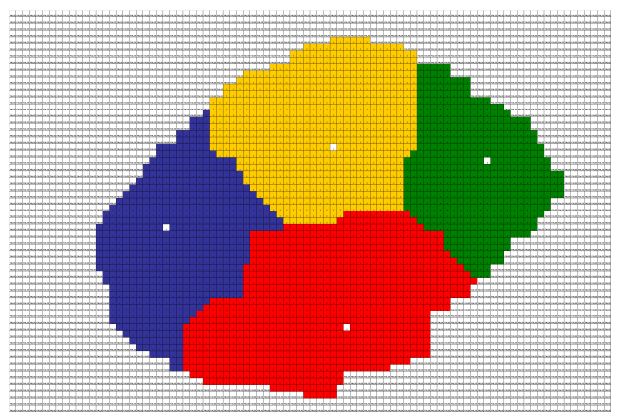

Fig. 9. Assigning the other cells to the located facilities.

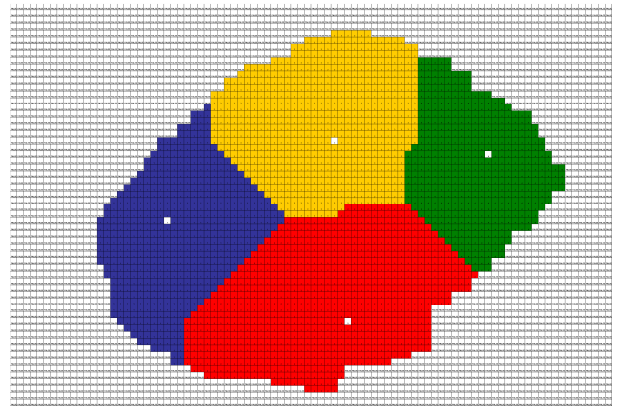

Fig. 10. Allocating all cells to the most suitable located facility. 
Table I Sample data of functions.

\begin{tabular}{|c|l|l|l|l|l|l|}
\hline$k$ & $c$ & $r$ & $D$ & $F$ & $O$ & $T$ \\
\hline 1 & $c_{1}$ & $r_{1}$ & $D_{1}$ & $F_{1}$ & $O_{1}$ & $T_{1}$ \\
\hline 2 & $c_{2}$ & $r_{2}$ & $D_{2}$ & $F_{2}$ & $O_{2}$ & $T_{2}$ \\
\hline$\vdots$ & $\vdots$ & $\vdots$ & $\vdots$ & $\vdots$ & $\vdots$ & $\vdots$ \\
\hline$n$ & $c_{n}$ & $r_{n}$ & $D_{n}$ & $F_{n}$ & $O_{n}$ & $T_{n}$ \\
\hline
\end{tabular}

Table II Numerical input-output data for region $M$.

\begin{tabular}{|c|c|c|c|c|c|c|c|c|c|c|c|c|c|c|c|c|c|}
\hline$\vec{k}$ & $\bar{c}$ & $r$ & $D$ & $F$ & $O$ & $k$ & $\bar{c}$ & $r$ & $\bar{D}$ & $F$ & $O$ & $k$ & $\bar{c}$ & $r$ & $\bar{D}$ & $F$ & $O$ \\
\hline 1 & 15 & 20 & 524 & 6644 & 0.205 & 37 & 40 & 10 & 360 & 5955 & 0.204 & 73 & 55 & 35 & 580 & 5929 & 0.208 \\
\hline 2 & 15 & 25 & 520 & 6674 & 0.209 & 38 & 40 & 15 & 383 & 5973 & 0.202 & 74 & 55 & 40 & 696 & 6057 & 0.211 \\
\hline 3 & 15 & 30 & 516 & 6736 & 0.212 & 39 & 40 & 20 & 400 & 5989 & 0.200 & 75 & 55 & 45 & 750 & 6186 & 0.214 \\
\hline 4 & 20 & 10 & 518 & 6488 & 0.208 & 40 & 40 & 25 & 413 & 6004 & 0.203 & 76 & 55 & 50 & 792 & 6399 & 0.219 \\
\hline 5 & 20 & 15 & 512 & 6516 & 0.205 & 41 & 40 & 30 & 442 & 6093 & 0.207 & 77 & 55 & 55 & 800 & 6718 & 0.226 \\
\hline 6 & 20 & 20 & 508 & 6544 & 0.204 & 42 & 40 & 35 & 448 & 6173 & 0.210 & 78 & 60 & 15 & 263 & 5584 & 0.199 \\
\hline 7 & 20 & 25 & 502 & 6570 & 0.207 & 43 & 40 & 40 & 455 & 6255 & 0.213 & 79 & 60 & 20 & 371 & 5632 & 0.198 \\
\hline 8 & 20 & 30 & 489 & 6634 & 0.211 & 44 & 40 & 45 & 500 & 6340 & 0.217 & 80 & 60 & 25 & 600 & 5672 & 0.201 \\
\hline 9 & 20 & 35 & 482 & 6656 & 0.214 & 45 & 40 & 50 & 504 & 6523 & 0.221 & 81 & 60 & 30 & 686 & 5781 & 0.204 \\
\hline 10 & 25 & 10 & 516 & 6357 & 0.206 & 46 & 45 & 5 & 280 & 5666 & 0.207 & 82 & 60 & 35 & 720 & 5921 & 0.207 \\
\hline 11 & 25 & 15 & 508 & 6381 & 0.204 & 47 & 45 & 10 & 298 & 5818 & 0.203 & 83 & 60 & 40 & 738 & 6063 & 0.210 \\
\hline 12 & 25 & 20 & 497 & 6403 & 0.203 & 48 & 45 & 15 & 320 & 5837 & 0.201 & 84 & 60 & 45 & 750 & 6208 & 0.214 \\
\hline 13 & 25 & 25 & 488 & 6425 & 0.206 & 49 & 45 & 20 & 360 & 5854 & 0.199 & 85 & 60 & 50 & 750 & 6432 & 0.218 \\
\hline 14 & 25 & 30 & 495 & 6498 & 0.209 & 50 & 45 & 25 & 400 & 5870 & 0.203 & 86 & 65 & 20 & 440 & 5582 & 0.196 \\
\hline 15 & 25 & 35 & 449 & 6534 & 0.213 & 51 & 45 & 30 & 461 & 5958 & 0.206 & 87 & 65 & 25 & 600 & 5626 & 0.199 \\
\hline 16 & 25 & 40 & 398 & 6572 & 0.216 & 52 & 45 & 35 & 500 & 6054 & 0.209 & 88 & 65 & 30 & 640 & 5732 & 0.203 \\
\hline 17 & 30 & 5 & 512 & 6026 & 0.209 & 53 & 45 & 40 & 534 & 6152 & 0.212 & 89 & 65 & 35 & 667 & 5868 & 0.206 \\
\hline 18 & 30 & 10 & 506 & 6224 & 0.206 & 54 & 45 & 45 & 547 & 6253 & 0.216 & 90 & 65 & 40 & 686 & 6010 & 0.209 \\
\hline 19 & 30 & 15 & 497 & 6245 & 0.203 & 55 & 45 & 50 & 575 & 6445 & 0.220 & 91 & 65 & 45 & 700 & 6155 & 0.212 \\
\hline 20 & 30 & 20 & 480 & 6264 & 0.202 & 56 & 45 & 55 & 600 & 6760 & 0.227 & 92 & 65 & 50 & 702 & 6369 & 0.217 \\
\hline 21 & 30 & 25 & 467 & 6282 & 0.205 & 57 & 50 & 5 & 202 & 5552 & 0.206 & 93 & 70 & 20 & 477 & 5524 & 0.195 \\
\hline 22 & 30 & 30 & 476 & 6363 & 0.208 & 58 & 50 & 10 & 218 & 5680 & 0.203 & 94 & 70 & 25 & 600 & 5572 & 0.198 \\
\hline 23 & 30 & 35 & 440 & 6413 & 0.212 & 59 & 50 & 15 & 232 & 5701 & 0.200 & 95 & 70 & 30 & 615 & 5673 & 0.201 \\
\hline 24 & 30 & 40 & 396 & 6465 & 0.215 & 60 & 50 & 20 & 280 & 5720 & 0.199 & 96 & 70 & 35 & 629 & 5805 & 0.204 \\
\hline 25 & 30 & 45 & 400 & 6519 & 0.219 & 61 & 50 & 25 & 360 & 5738 & 0.202 & 97 & 70 & 40 & 640 & 5944 & 0.207 \\
\hline 26 & 35 & 5 & 490 & 5903 & 0.208 & 62 & 50 & 30 & 507 & 5823 & 0.205 & 98 & 70 & 45 & 650 & 6090 & 0.211 \\
\hline 27 & 35 & 10 & 483 & 6090 & 0.205 & 63 & 50 & 35 & 587 & 5936 & 0.208 & 99 & 75 & 25 & 504 & 5507 & 0.196 \\
\hline 28 & 35 & 15 & 476 & 6109 & 0.202 & 64 & 50 & 40 & 635 & 6051 & 0.211 & 100 & 75 & 30 & 515 & 5602 & 0.199 \\
\hline 29 & 35 & 20 & 470 & 6126 & 0.201 & 65 & 50 & 45 & 640 & 6168 & 0.215 & 101 & 75 & 35 & 520 & 5727 & 0.202 \\
\hline
\end{tabular}




\begin{tabular}{|l|l|l|l|l|l|l|l|l|l|l|l|l|l|l|l|l|l|}
30 & 35 & 25 & 462 & 6142 & 0.204 & 66 & 50 & 50 & 664 & 6370 & 0.220 & 102 & 75 & 40 & 535 & 5862 & 0.205 \\
\hline 31 & 35 & 30 & 451 & 6228 & 0.207 & 67 & 50 & 55 & 700 & 6696 & 0.226 & 103 & 75 & 45 & 557 & 6006 & 0.209 \\
\hline 32 & 35 & 35 & 424 & 6292 & 0.211 & 68 & 55 & 10 & 200 & 5616 & 0.202 & 104 & 80 & 30 & 582 & 5515 & 0.197 \\
\hline 33 & 35 & 40 & 394 & 6359 & 0.214 & 69 & 55 & 15 & 246 & 5651 & 0.200 & 105 & 80 & 35 & 619 & 5630 & 0.200 \\
\hline 34 & 35 & 45 & 400 & 6428 & 0.218 & 70 & 55 & 20 & 280 & 5682 & 0.198 & 106 & 80 & 40 & 662 & 5756 & 0.203 \\
\hline 35 & 35 & 50 & 412 & 6603 & 0.222 & 71 & 55 & 25 & 360 & 5708 & 0.201 & & & & & & \\
\hline 36 & 40 & 5 & 377 & 5783 & 0.207 & 72 & 55 & 30 & 493 & 5804 & 0.204 & & & & & & \\
\hline
\end{tabular}

Table III Mean and standard deviation of each dimension.

\begin{tabular}{|l|l|l|l|l|l|}
\hline & $c$ & $r$ & $D$ & $F$ & $O$ \\
\hline$m^{\prime}$ & 46 & 29 & 503 & 6090 & 0.207 \\
\hline$s^{\prime}$ & 14 & 11 & 103 & 298 & 0.006 \\
\hline
\end{tabular}

Table IV Center of each cluster in both the scalelessed dimensions and the rescalled dimensions in the FRB with the output $D$.

\begin{tabular}{|l|l|l|l|l|l|l|}
\hline Cluster & $c_{\text {Scalelessed }}$ & $r_{\text {Scalelessed }}$ & $D_{\text {Scalelessed }}$ & $c$ & $r$ & $D$ \\
\hline 1 & -1.143 & -0.364 & -0.35 & 29.998 & 24.996 & 466.950 \\
\hline 2 & 1.357 & 1 & 1.777 & 64.998 & 40 & 686.031 \\
\hline 3 & -0.429 & 1 & -0.466 & 39.994 & 40 & 455.002 \\
\hline 4 & -0.071 & -1.273 & -1.777 & 45.006 & 14.997 & 319.969 \\
\hline 5 & -1.5 & -1.727 & 0.126 & 25 & 10.003 & 515.978 \\
\hline 6 & 1.714 & -0.364 & 0.942 & 69.996 & 24.996 & 600.026 \\
\hline 7 & 0.286 & 1.909 & 1.563 & 50.004 & 49.999 & 663.989 \\
\hline
\end{tabular}

Table V Center of each cluster in both the scalelessed dimensions and the rescalled dimensions in the FRB with the output $F$.

\begin{tabular}{|l|l|l|l|l|l|l|}
\hline Cluster & $c_{\text {Scalelessed }}$ & $r_{\text {Scalelessed }}$ & $F_{\text {Scalelessed }}$ & $c$ & $r$ & $F$ \\
\hline 1 & 0.643 & -0.364 & -1.281 & 55.002 & 24.996 & 5708.262 \\
\hline 2 & -0.071 & 1 & 0.209 & 45.006 & 40.000 & 6152.282 \\
\hline 3 & -1.5 & -0.364 & 1.122 & 25 & 24.996 & 6424.356 \\
\hline 4 & -0.429 & -1.273 & -0.393 & 39.994 & 14.997 & 5972.886 \\
\hline 5 & 1.714 & 1 & -0.49 & 69.996 & 40.000 & 5943.980 \\
\hline 6 & -1.143 & 1.455 & 1.439 & 29.998 & 45.005 & 6518.822 \\
\hline 7 & 2.071 & 0.091 & -1.638 & 74.994 & 30.001 & 5601.876 \\
\hline 8 & 0.64 & 1.91 & 1.04 & 55.002 & 49.999 & 6398.430 \\
\hline 9 & 0.29 & -1.73 & -1.38 & 50.004 & 10.003 & 5679.654 \\
\hline 10 & -1.50 & -1.73 & 0.90 & 25 & 10.003 & 6356.710 \\
\hline 11 & -0.07 & 2.36 & 2.25 & 45.006 & 55.004 & 6759.904 \\
\hline
\end{tabular}


Table VI Center of each cluster in both the scalelessed dimensions and the rescalled dimensions in the

FRB with the output $O$.

\begin{tabular}{|l|l|l|l|l|l|l|}
\hline Cluster & $c_{\text {Scalelessed }}$ & $r_{\text {Scalelessed }}$ & $O_{\text {Scalelessed }}$ & $l$ & $r$ & $O$ \\
\hline 1 & -0.429 & -1.273 & -0.882 & 39.994 & 14.997 & 0.202 \\
\hline 2 & 0.643 & 0.545 & 0.083 & 55.002 & 34.995 & 0.207 \\
\hline 3 & -1.143 & 0.545 & 0.778 & 29.998 & 34.995 & 0.212 \\
\hline 4 & 1.357 & -0.364 & -1.262 & 64.998 & 24.996 & 0.199 \\
\hline 5 & 0.286 & 1.909 & 2.117 & 50.004 & 49.999 & 0.220 \\
\hline 6 & -1.857 & -0.818 & -0.542 & 20.002 & 20.002 & 0.204 \\
\hline 7 & 2.071 & 1 & -0.333 & 74.994 & 40 & 0.205 \\
\hline 8 & -1.14 & -2.18 & 0.28 & 29.998 & 4.998 & 0.209 \\
\hline
\end{tabular}

Table VII Parameters of the FRB with the output $D$.

\begin{tabular}{|l|l|l|l|l|l|l|l|}
\hline Rule & $m_{c}$ & $s_{c}$ & $m_{r}$ & $s_{r}$ & $a$ & $b$ & $c$ \\
\hline 1 & 29.998 & 6.196 & 24.996 & 5.652 & -2.905 & -0.204 & 565.297 \\
\hline 2 & 64.998 & 6.585 & 40 & 5.706 & -5.524 & 4.296 & 835.875 \\
\hline 3 & 39.994 & 6.026 & 40 & 5.706 & 7.890 & 1.254 & 93.473 \\
\hline 4 & 45.006 & 6.333 & 14.997 & 5.716 & -12.416 & 4.048 & 815.086 \\
\hline 5 & 25 & 6.120 & 10.003 & 5.203 & 0.028 & -0.029 & 515.615 \\
\hline 6 & 69.996 & 6.728 & 24.996 & 5.652 & -1.431 & 13.931 & 284.182 \\
\hline 7 & 50.004 & 5.586 & 49.999 & 5.191 & 17.212 & 1.976 & -272.632 \\
\hline
\end{tabular}

Table VIII Parameters of the FRB with the output $F$.

\begin{tabular}{|l|l|l|l|l|l|l|l|}
\hline Rule & $m_{c}$ & $s_{c}$ & $m_{r}$ & $s_{r}$ & $a$ & $b$ & $c$ \\
\hline 1 & 55.002 & 5.951 & 24.996 & 5.360 & -6.826 & 16.440 & 5681.322 \\
\hline 2 & 45.006 & 6.303 & 40.000 & 5.222 & -16.347 & 17.857 & 6203.764 \\
\hline 3 & 25 & 6.256 & 24.996 & 5.360 & -24.920 & 15.709 & 6648.176 \\
\hline 4 & 39.994 & 5.376 & 14.997 & 5.232 & -20.949 & 0.683 & 6846.980 \\
\hline 5 & 69.996 & 5.356 & 40.000 & 5.222 & -15.600 & 23.087 & 6129.675 \\
\hline 6 & 29.998 & 5.646 & 45.005 & 5.244 & -16.105 & 14.522 & 6371.920 \\
\hline 7 & 74.994 & 5.496 & 30.001 & 4.848 & -12.472 & 15.456 & 6060.894 \\
\hline 8 & 55.002 & 5.951 & 49.999 & 5.213 & -4.219 & 47.194 & 4360.021 \\
\hline 9 & 50.004 & 6.212 & 10.003 & 5.489 & -8.873 & 17.464 & 5918.009 \\
\hline 10 & 25 & 6.256 & 10.003 & 5.489 & -30.346 & 25.433 & 6827.965 \\
\hline 11 & 45.006 & 6.303 & 55.004 & 6.013 & -25.674 & 72.049 & 3981.581 \\
\hline
\end{tabular}

Table IX Parameters of the FRB with the output $O$.

\begin{tabular}{|l|l|l|l|l|l|l|l|}
\hline Rule & $m_{c}$ & $s_{c}$ & $m_{r}$ & $s_{r}$ & $a$ & $b$ & $c$ \\
\hline 1 & 39.994 & 5.086 & 14.997 & 5.135 & -0.00004 & -0.00028 & 0.20779 \\
\hline 2 & 55.002 & 5.611 & 34.995 & 5.809 & -0.00017 & 0.00068 & 0.19317 \\
\hline 3 & 29.998 & 6.021 & 34.995 & 5.809 & -0.00019 & 0.00063 & 0.19586 \\
\hline 4 & 64.998 & 5.437 & 24.996 & 5.054 & -0.00032 & 0.00044 & 0.20948 \\
\hline 5 & 50.004 & 5.368 & 49.999 & 4.719 & -0.00015 & 0.00114 & 0.17075 \\
\hline 6 & 20.002 & 4.932 & 20.002 & 5.395 & -0.00031 & 0.00030 & 0.20576 \\
\hline 7 & 74.994 & 5.121 & 40 & 5.374 & -0.00041 & 0.00064 & 0.21082 \\
\hline 8 & 29.998 & 6.021 & 4.998 & 5.014 & -0.00016 & -0.00044 & 0.21664 \\
\hline
\end{tabular}

